\title{
La demora de la colonoscopía luego de un resultado positivo de una prueba de sangre oculta en materia fecal se asocia a mayor probabilidad de malignidad y enfermedad avanzada
}

Delayed colonoscopy following a possitive faecal occult blood test is associated with a higher chance of malignancy and advanced disease

\section{Comentado de:}

Corley DA, et al. JAMA. 2017 Apr 25;317(16):1631-1641. doi: 10.1001/jama.2017.3634 ${ }^{1}$.

\section{Objetivo}

Evaluar si el tiempo a la videocolonoscopía (VCC) luego de una prueba de sangre oculta en materia fecal inmunológica (SOMFi) realizada como tamizaje de cáncer colorrectal (CCR) se asocia con una mayor probabilidad de desarrollar esta enfermedad $y / 0$ de presentar un estadio más avanzado al momento del diagnóstico.

\section{Diseño y lugar}

Cohorte retrospectiva (Enero de 2010 a Diciembre de 2014) que incluyó afiliados a una empresa de medicina prepaga de los EE.UU. (Kaiser Permanente).

\section{Pacientes}

Fueron incluidos pacientes de 50 a 70 años de edad con un resultado positivo de SOMFi, que habían realizado una colonoscopía de seguimiento. Criterios de exclusión: historia previa de CCR, menos de un año de afiliación, VCC en los diez años previos o sigmoidoscopía en los cinco años previos a la prueba de $\mathrm{SOMFi}$, diagnóstico de CCR realizado durante los primeros siete días posteriores al resultado positivo de la SOMFi.

\section{Evaluación de factores pronósticos y medición de} resultados principales

La exposición fue el tiempo a la colonoscopía luego de un resultado positivo de SOMFi. Los resultados primarios fueron: cual- quier adenocarcinoma colorrectal diagnosticado en el momento de la VCC de seguimiento o hasta seis meses después de realizada esta, la incidencia de CCR avanzado (estadio III o IV, con compromiso linfático o metástasis a distancia, respectivamente) y de adenomas de alto grado (p. ej. adenomas tubulovellosos o vellosos). Todos los diagnósticos se obtuvieron de los registros de cáncer de Kaiser Permanente. Los resultados fueron ajustados por variables demográficas, clínicas y de uso de recursos sanitarios.

\section{Resultados principales}

De los 1.258 .039 pacientes de 50 a 75 años que realizaron el rastreo con SOMFi, $106.520(8,5 \%)$ tuvieron un resultado positivo. Luego de excluir a quienes cumplían alguno de los criterios preestablecidos, quedaron 81.518 pacientes. De estos, 70.124 $(86 \%)$ realizaron VCC de seguimiento al finalizar el estudio. Su mediana de edad fue 61 años (intervalo intercuartilo o IIC 55 a 67 $53 \%$ varones) y $42 \%$ tenía IMC mayor o igual a 30 . La mediana de tiempo a la colonoscopía fue 37 días (RIC, 23 a 62). Se diagnosticaron 2.191 casos de cualquier CCR y 601 casos de CCR avanzado.

Comparados con la realización de la VCC entre los 8 y 30 días luego del resultado positivo de la SOMFi (grupo de referencia), cada intervalo de 30 días adicional se asoció con un incremento de la probabilidad de $3 \%$ para cualquier CCR (OR, 1,03; IC $95 \%$, $1,03$ a 1,04$)$ y del $5 \%$ para el CCR avanzado (OR, 1,05 ; IC $95 \%$, $1,04$ a 1,06$)$. Los resultados principales se describen en la tabla 1.

Tabla 1. Probabilidad ajustada* de recibir un diagnóstico de cáncer colorrectal durante los primeros seis meses posteriores al resultado positivo de la prueba de sangre oculta en la materia fecal de acuerdo al tiempo transcurrido entre dicho resultado y la realización de la videocolonoscopía.

\begin{tabular}{|l|c|c|c|}
\hline $\begin{array}{l}\text { Tiempo desde el resultado positi- } \\
\text { vo en la SOMFi hasta la realiza- } \\
\text { ción de la VCC }\end{array}$ & $\begin{array}{c}\text { CCR } \\
\text { OR (IC 95 \%) }\end{array}$ & $\begin{array}{c}\text { CCR avanzado } \\
\text { OR (IC 95\%) }\end{array}$ & $\begin{array}{c}\text { Adenomas } \\
\text { de alto grado } \\
\text { OR (IC 95\%) }\end{array}$ \\
\hline Durante los primeros 30 días & 1 & 1 & 1 \\
\hline 30 días a 12 meses & $1,37(1,09$ a 1,70) & $1,55(1,05$ a 2,28) & $1,07(0,92$ a 1,26$)$ \\
\hline Más de 12 meses & $2,25(1,89$ a 2,68$)$ & $3,22(2,44$ a 4,25$)$ & $1,32(1,15$ a 1,52$)$ \\
\hline
\end{tabular}

${ }^{*}$ Ajustado porsexo, edad, etnia auto-reportada, índice de masa corporal (IMC), región, año de realización de la SOMFi, realización de rastreo previo con SOMFi, recepción de vacuna antigripal o antineumocócica, presencia de síntomas gastrointestinales (sangrados digestivos, pérdida de peso inexplicada, dolor abdominal, diarrea, diverticulitis, enfermedad inflamatoria intestinal o síndrome de Lynch), diagnóstico de anemia ferropénica o diabetes, estatus tabáquico, número de consultas de atención primaria y número de días de hospitalización. CCR: Cáncer colorrectal. OR: Odds Ratio. IC $95 \%$ : Intervalo de confianza del $95 \%$. VCC: videocolonoscopía. 


\section{Conclusiones}

Entre los pacientes con un resultado positivo de SOMFi, la realización tardía de la VCC se asoció con una mayor probabilidad de presentar CCR y una enfermedad de estadío más avanzado al momento del diagnóstico.

Fuente de financiamiento/conflicto de interés de los autores: financiado por el Instituto Nacional del Cáncer de los EE.UU (National Cancer Institute)

\section{Comentario}

El rastreo del CCR en hombres y mujeres de 50 a 75 años de edad es una práctica preventiva avalada por la evidencia aunque su implementación como una política sanitaria a nivel poblacional es variable. Se dispone de varias estrategias entre las que se incluyen la VCC cada diez años, la rectosigmoideoscopía cada cinco años combinada con detección anual de SOMFi, y la pesquisa con SOMF anual ${ }^{2,3}$. En el estudio resumido en este artículo, un seguro de salud privado de los EE.UU. implementa un programa de rastreo de CCR mediante el cual sus afiliados que no tienen actualizada esta práctica son convocados (personalmente o mediante correo postal) en forma sistemática cada año para que realicen una prueba diagnóstica sencilla, de bajo costo, con buena sensibilidad y especificidad: la SOMFi. Todo resultado positivo debe ser seguido de un estudio que evalúe la totalidad de la mucosa colónica (habitualmente VCC). Sin embargo, no todos los pacientes lleguen a realizar la evaluación colónica completa, lo que disminuiría el impacto del tamizaje con SOMFi.

Este estudio documentó que en un sistema cerrado de salud que cuenta con un programa de búsqueda activa de pacientes elegibles, los resultados fueron similares a los publicados por otros autores respecto de intervenciones a nivel poblacional ${ }^{4}$. Por otro lado, a medida que se incrementa el tiempo transcurrido entre el resultado positivo de la SOMFi y la VCC (en especial luego de 12 meses), se incrementa la probabilidad de detectar lesiones de peor pronóstico, lo que no resulta demasiado novedoso ya que está en sintonía con la recomendación de reiterar la SOMFi anualmente luego de un resultado negativo. Sin embargo, este aumento de riesgo comienza a evidenciarse ya a los seis meses de un resultado positivo de la SOMFi, lo que destaca la importancia de optimizar la accesibilidad a la VCC en las personas incluidas en programas de tamizaje.

\section{Conclusiones de la comentadora}

Es importante que los pacientes con resultados positivos a la SOMFi tengan acceso oportuno una evaluación completa de su mucosa colónica.

Vietto Valeria [ Servicio de Medicina Familiar y Comunitaria, Hospital Italiano de Buenos Aires valeria.vietto@hospitalitaliano.org.ar ]

Vietto V. La demora de la colonoscopía luego de un resultado positivo de una prueba de sangre oculta en materia fecal se asocia a mayor probabilidad de malignidad y enfermedad avanzada Evid Actual Pract Ambul. 2019;22(1):e001078. Comentado de: Corley DA, Jensen CD, Quinn VP, Doubeni CA, Zauber AG, Lee JK et al. Association Between Time to Colonoscopy After a Positive Fecal Test Result and Risk of Colorectal Cancer and Cancer Stage at Diagnosis. JAMA. 2017 Apr 25;317(16):1631-1641. PMID: 28444278

\section{Referencias}

1. Corley DA, Jensen CD, Quinn VP, Doubeni CA, Zauber AG, Lee JK, et al. Association between time to colonoscopy after a positive fecal test result and risk of colorectal Cancer and Cancer stage at Diagnosis. JAMA - Journal of the American Medical Association. 2017;null. Available from: 10.1001/jama.2017.3634.

2. Fenocchi E, Martínez L, Tolve J, Montano D, Rondán M, Parra-Blanco A, et al. Screening for colorectal cancer in Uruguay with an immunochemical faecal occult blood test. European journal of cancer prevention : the official journal of the European Cancer Prevention Organisation (ECP). 2006 oct;15(5):384-90.

3. Lin JS, Piper MA, Perdue LA, Rutter CM, Webber EM, O’Connor E, et al. Screening for Colorectal Cancer. JAMA. 2016 jun;315(23):2576. Available from: 10.1001/jama.2016.3332.

4. Bibbins-Domingo K, Grossman DC, Curry SJ, Davidson KW, Epling JW, García FAR, et al. Screening for Colorectal Cancer. JAMA. 2016 jun;315(23):2564. Available from: 10.1001/jama.2016.5989. 